\title{
MULTIPLE POINTS OF A RANDOM FIELD
}

\author{
NARN RUEIH SHIEH ${ }^{1}$
}

\begin{abstract}
We prove that a $d$-dimensional random field $X \equiv\{X(t)\}_{t \in R_{+}^{N}}$ has uncountably many $r$-multiple points a.s. when it satisfies Pitt's $\left(A_{r}\right)$ condition [9]. Those $t$ 's for which $X(t)$ hits the multiple point can be separated by any given positive number, and multiple points can occur while $t$ is restricted to any given "time inteval". Two corollaries concerning Gaussian fields and fields with independent increments are also presented.
\end{abstract}

1. Introduction. In this paper, we shall establish a new connection between local times and multiple points of a random field. Roughly speaking, our result shows that a random field has uncountably many $r$-multiple points a.s. when it has $L^{r}$ local times a.s. We say that a point $x \in R^{d}$ is an $r$-multiple point of a function $X: R_{+}^{N} \rightarrow R^{d}$ if there exists distinct $t_{1}, \ldots, t_{t}$ such that

$$
x=X\left(t_{1}\right)=\cdots=X\left(t_{r}\right) .
$$

Assume that $X \equiv\{X(t)\}_{t \in R_{+}^{N}}$ is an $(N, d)$ random field, i.e., a family of $R^{d}$-valued random variables parametrized by $t \in R_{+}^{N}$. We like to determine whether for almost all $\omega, X(\cdot, \omega)$ has at least one $r$-multiple point, for given $r \geq 2$. This selfintersection problem has received the attention of various authors over the years. It was solved for one-parameter Brownian motions by Dvoretzky et al., which was also extended to processes with independent increments. A recent paper in this area with a satisfactorily complete bibliography is that of Hendricks [7]. On the other hand, recent works of Kono [8], Goldman [6], Cuzick [4] and Rosen [10] concern this problem for Gaussian random fields. Our current work is related to them, in which local time theory plays a dominant role. An excellent survey on local times is that of Geman and Horowitz [5]. Let us state our main result. Assume that $X=\{X(t)\}$ is an $(N, d)$ random field. For an integer $k \geq 1$, let $t_{1}, \ldots, t_{k}$ be arbitrary distinct points in $R_{+}^{N}$, and $x_{1}, \ldots, x_{k}$ arbitrary points in $R^{d}$. Put $\bar{t}=\left(t_{1}, \ldots, t_{k}\right), \bar{x}=\left(x_{1}, \downarrow ., x_{k}\right)$, and let $p_{k}(\bar{x}, \bar{t})$ be the joint density function of $X\left(t_{1}\right), \ldots, X\left(t_{k}\right)$ at $\bar{x}$, where the latter density is assumed to exist. ${ }^{1}$

THEOREM 1. For some given integer $r \geq 2$ suppose that the $p_{r}(\cdot, \cdot)$ of $X$ satisfies the following Pitt's condition (Pitt [9]):

There exists a function $g_{r}(\cdot)$ such that

(i) $p_{r}(\bar{x}, \bar{t}) \leq g_{r}(\bar{t})$ for almost all $\bar{x}$,

(ii) $\int_{I^{r}} g_{r}(\bar{t}) d \bar{t}<\infty$ for every compact "box" $I \subset R_{+}^{N}$.

Received by the editors August 1, 1983 and, in revised form, October 22, 1983.

1980 Mathematics Subject Classification. Primary 60G17, 60G60.

Key words and phrases. Multiple points, local times, random fields, Pitt's condition.

${ }^{1}$ This work is supported in part by the National Science Council, Taiwan, China.

(C) 1984 American Mathematical Society $0002-9939 / 84 \$ 1.00+\$ .25$ per page 
Then, $X$ has uncountably many $r$-multiple points along almost all paths. Moreover, for each given $h>0$, we may require that $t_{1}, \ldots, t_{r}$ in (1.1) satisfy $\left|t_{j}-t_{j-1}\right| \geq$ $h, j=1, \ldots, r, t_{0}=0$.

Furthermore, some minor modification on the proof of Theorem 1 yields the following

THEOREM 2. For any given $a, b \in R^{N}, 0 \leq a<b$ (coordinatewise), under the hypotheses of Theorem $1, X$ has uncountably many $r$-multiple points along almost all paths while the "time" parameter $t$ belongs to $[a, b]$ only.

Our proof of Theorem 1 is related to the arguments in Berman $[\mathbf{1}, \mathbf{2}]$. It should be mentioned that Berman's request in [2] only works for the case $r$ being a power of 2 , and is based on the existence of local times which belong to $L^{r}\left(R^{d} \times \Omega\right)$. However, our results in this paper, assuming Pitt's condition, work for any integer $r \geq 2$ and, in fact, rely only on local times which are a.s. "locally" $L^{r}$-integrable. See also the Remark at the end of $\S 2$.

The author is grateful to Professor S. M. Berman for giving him valuable preprints and discussions on local time theory. Furthermore, the advice from the referee is sincerely appreciated.

2. The proof of Theorems 1 and 2. We proceed in four steps.

$1^{\circ}$. For each $n=1,2, \ldots$, and $\bar{i}=\left(i_{1}, \ldots, i_{N}\right), i_{j} \in\left\{1, \ldots, 2^{n}\right\}$, define

$$
J_{n, \bar{i}}=\left(i_{1} h, \ldots, i_{N} h\right)+\prod_{j=1}^{N}\left[\frac{i_{j}-1}{2^{n}}, \frac{i_{j}}{2^{n}}\right] \text {. }
$$

Put $I_{n}=\bigcup_{\bar{i}} J_{n, \bar{i}}$. According to Pitt $[9$, Theorem 3.1], for almost all $\omega, X(\cdot, \omega)$ has a local time $\alpha_{n}(x) \equiv \alpha\left(x, \omega, I_{n}\right)$ over $I_{n}$ and $\alpha_{n}(x)$ can be chosen to be jointly measurable in $(x, \omega)$ and satisfying $E \int_{K} \alpha_{n}^{r}(x) d x<\infty$ for each compact $K \subset R^{d}$.

$2^{\circ}$. Since $\alpha_{n}(x)=\sum_{\bar{I}} \alpha\left(x, J_{n, \bar{i}}\right)$, we may write $\left(\alpha_{n}(x)\right)^{r}=A_{n}(x)+B_{n}(x)$, where $A_{n}(x) \equiv A_{n}(x, \omega)$ and $B_{n}(x) \equiv B_{n}(x, \omega)$ are defined, respectively, by the sums of those $\left(\prod_{k=1}^{r} \alpha\left(x, \omega, J_{n, \bar{i}(k)}\right)\right)$ 's in which the indices $(\bar{i}(1), \ldots, \bar{i}(r))$ are all distinct and are not all distinct. We claim that $\lim _{n \rightarrow \infty} E \int_{K} B_{n}(x) d x=0$ for each compact $K \subset R^{d}$. Set $\Lambda_{n}=\bigcup\left\{\prod_{k=1}^{r} J_{n, \bar{i}(k)}: \bar{i}(1), \ldots, \bar{i}(r)\right.$ are not all distinct $\}$. Then by the Fubini Theorem

$$
\begin{aligned}
E \int_{K} B_{n}(x) d x & =\int_{K} \int_{\Lambda_{n}} p_{r}(\underbrace{x, \ldots, x}_{r \text { terms }}, \bar{t}) d \bar{t} d x \\
& \leq \operatorname{Leb}_{R^{d}}(K) \int_{\Lambda_{n}} g_{r}(\bar{t}) d \bar{t} .
\end{aligned}
$$

Since $\lim _{n \rightarrow \infty} \operatorname{Leb}_{R^{N r}}\left(\Lambda_{n}\right)=0$, by Pitt's condition (ii),

$$
\lim _{n \rightarrow \infty} \int_{\Lambda_{n}} g_{r}(\bar{t}) d \bar{t}=0
$$

$3^{\circ}$. Using a diagonal argument, we can find a subsequence $\left\{n_{k}\right\}$ and a sequence of compact sets $K_{l}$ increasing to $R^{d}$ such that

$$
\lim _{n_{k} \rightarrow \infty} \int_{K_{l}} B_{n_{k}}(x, \omega) d x=0
$$


for all $l$ and all $\omega \notin E, E$ a set of probability zero. For each $\omega \notin E$, we can choose a large compact set $K=K(\omega)$ (one of $\left\{K_{l}\right\}$ above) and a large integer $n=n(\omega)$ such that $\int_{K} A_{n}(x, \omega) d x>0$. Then there exists at least one term in $A_{n}(x, \omega)$, say $\prod_{k=1}^{r} \alpha\left(x, \omega, J_{n, \bar{i}(k)}\right)$, such that $\int_{K} \prod_{k=1}^{r} \alpha\left(x, \omega, J_{n, \bar{i}(k)}\right) d x>0$. Therefore, the set $\bigcap_{k=1}^{r} X\left(J_{n, \bar{i}(k)} ; \omega\right)$ must be of positive $d$-dimensional Lebesgue measure, and thus this set contains uncountably many points. Since $\bar{i}(1), \ldots, \bar{i}(r)$ are all distinct and the distance between different $J_{n, \bar{i}(k)}$ 's is at least $h$, all the points in $\bigcap_{k=1}^{r} X\left(J_{n, \bar{i}(k)} ; \omega\right)$ are our desired multiple points. This completes the proof of Theorem 1 .

$4^{\circ}$. To prove Theorem 2 , instead of fixed $h>0$ and intervals of length $1 / 2^{n}$, we choose appropriate $h_{n}$ and $2^{n}$ subintervals of $\left[a_{j}, b_{j}\right]$ such that the total lengths of these subintervals plus $2^{n} h_{n}$ are equal to $b_{j}-a_{j}$. Define

$$
\left.J_{n, \bar{i}}=\left(i_{1} h_{n}, \ldots, i_{N} h_{n}\right)+\prod_{j=1}^{N} \text { (subintervals of }\left[a_{j}, b_{j}\right]\right) .
$$

Then, the rest of the arguments follow as above.

REMARKS. As one can see from the proof given above, we have in fact proved the existence of multiple images (see Berman [2]) which are separated by a preassigned $h$. We prefer to state our results in terms of multiple points since it seems that the latter is a more prevailing concept.

3. Corollaries. First, we assume that $X \equiv\{X(t)\}$ is an $(N, d)$ mean zero, stochastically continuous Gaussian field. Let $D_{k}(\bar{t})$ denote the determinant of the covariance matrix of $X\left(t_{1}\right), \ldots, X\left(t_{k}\right)$. Then, the following corollary follows immediately from Theorem 1 and the fact that the joint density $p_{r}(\bar{t}, \bar{x})$ is dominated by $(2 \pi)^{-d r / 2} D_{r}^{-1 / 2}(\bar{t})$.

COROLlaRY 1. If $\int_{I^{r}} D_{r}^{-1 / 2}(\bar{t}) d \bar{t}<\infty$ for every box $I \subset R_{+}^{N}$, then $X$ has uncountably many $r$-multiple points along almost all paths.

Next, we assume that $X$ is an $(N, d)$ field with stationary, independent $N$ parameter increments. Assume further that $X$ is stochastically continuous and that $X(t)=0$ if one component of $t$ is zero. Then, as the one-parameter case, there exists an infinitely divisible law $\psi$ on $R^{d}$ such that $E\left(e^{i u \cdot X(t)}\right)=e^{-\left(\prod_{j=1}^{N} t_{j}\right) \psi(u)}$ for $u \in R^{d}$ and $t=\left(t_{1}, \ldots, t_{N}\right)$. Recall that the lower index $\beta$ of $\psi$ defined by Blumenthal and Getoor $[3]$ is equal to $\beta=\sup \left\{\alpha \geq 0:|u|^{-\alpha} \operatorname{Re} \psi(u) \rightarrow \infty\right.$ as $\left.|u| \rightarrow \infty\right\}$.

COROLlARY 2. If $d<\beta N$, then for any given $r \geq 2, X$ has uncountably many $r$-multiple points along almost all paths.

Proof. In [11, Theorem B], we have shown that if $d<\beta N$, then there exist a $\delta: 0<\delta<1$, such that for every positive even integer $k$ and every compact box $I \subset R_{+}^{N}$ bounded away from 0

$$
\int_{I^{k}}\left\{\int_{\left(R^{d}\right)^{k}}\left|E e^{i \sum_{j=1}^{k} u_{j} \cdot X\left(t_{j}\right)}\right| \prod_{j=1}^{k}\left|u_{j}\right|^{\delta} d u_{1} \cdots d u_{k}\right\} d t_{1} \cdots d t_{k}<\infty .
$$

By the Fourier Inversion Theorem, we see that Pitt's condition actually holds for any given $r \geq 2$ when $d<\beta N$. 
In the case that $X$ is an index $\beta$ field (including Lévy's multiparameter Brownian motion) or a Brownian sheet (see [5] for definitions), more results about the existence and nonexistence of multiple points of $X$ have been recently obtained by Cuzick [4] and Rosen [10]. However, their results, in general, concern only "with positive probability".

\section{REFERENCES}

1. S. M. Berman, Local nondeterminism and local times of general stochastic processes, preprint.

2. __ Multiple images of stochastic processes, preprint.

3. R. M. Blumenthal and R. K. Getoor, Sample functions of stochastic processes with stationary independent increments, J. Math. Mech. 10 (1961), 493-516.

4. J. Cuzick, Multiple points of a Gaussian vector field, Z. Wahrsch. Verw. Gebiete 61 (1982), 431-436.

5. D. Geman and J. Horowitz, Occupation densities, Ann. Probab. 8 (1980), 1-67.

6. A. Goldman, Points multiples des trajectoires de processus Gaussians, Z. Wahrsch. Verw. Gebiete 57 (1981), 481-494.

7. W. J. Hendricks, Multiple points for transient symmetric Lévy processes in $R^{d}, \mathrm{Z}$. Wahrsch. Verw. Gebiete 49 (1979), 13-21.

8. N. Kono, Double points of a Gaussian sample path, Z. Wahrsch. Verw. Gebiete 45 (1978), 175180.

9. L. D. Pitt, Local times for Gaussian vector fields, Indiana Univ. Math. J. 27 (1978), 309-390.

10. J. Rosen, Self-intersections of random fields, preprint.

11. N. R. Shieh, Joint continuity of local times for random fields, preprint.

Department of Mathematics, National Taiwan University, Taipei, Taiwan, CHINA 